\title{
Cuestiones relevantes para la enseñanza de los tiempos pretéritos castellanos a hablantes polacos
}

\author{
Irene GARCÍA-OCHOA ROJAS \\ Universidad Complutense de Madrid \\ ijzus@hotmail.com
}

Recibido: Septiembre de 2010

Aceptado: Enero de 2011

\section{Resumen}

El artículo presenta los problemas más comunes que encuentran los estudiantes polacos en la lengua española, particularmente en el verbo. Para poder enseñar efectiva y claramente el español a hablantes polacos, no podemos tener ninguna duda sobre los conceptos que "tiempo" y "aspecto" tienen en las dos lenguas.

Palabras clave: enseñanza, español-polaco, pretérito, aspecto.

\section{Important Issues to Teach the Past Tense to Polish Students}

\begin{abstract}
This article presents the most common problems in the Spanish learning for Polish students, specially the verb. In order to teach effectively the Spanish language to Polish students, we should not have any doubt about the concepts of tense and aspect in both languages.
\end{abstract}

Key words: teaching, Spanish-Polish, past tense, aspect.

SUMARIO: 1. Introducción; 2. El aspecto: concepciones generales y formación en polaco; 3. Equivalencias y diferencias de expresión entre los sistemas verbales; 4. Conclusión; 5. Referencias bibliográficas. 


\section{Introducción}

El español se ha puesto de moda. Numerosos son los estudiantes de varios países que lo eligen como lengua extranjera, después del inglés, y muchos de ellos vienen a España para aprenderlo en una situación de inmersión lingüística.

Por otro lado, tenemos otro colectivo de extranjeros que se acercan al estudio del español por circunstancias laborales cuyos conocimientos previos de esta lengua difieren de los del primer grupo.

Tanto con el primer tipo de alumno como con el segundo, el profesor debe tener claro, cuando se trate del hablantes polacos, los conceptos de tiempo y aspecto en la lengua extranjera que enseña (español) como en la materna (polaco) de los estudiantes, para llevar a cabo con éxito la enseñanza del sistema verbal español.

\section{El aspecto: concepciones generales y formación en polaco}

En el español, como lengua romance, el tiempo es rasgo morfológico predominante a partir del cual se clasifican todas las formas. Sin embargo, en el polaco, como lengua eslava, se marcan morfológicamente tanto el tiempo como el aspecto, aunque predomina el aspecto ya que está presente también en las formas no personales.

En cuanto a lo que entendemos por aspecto en español la RAE (2005: 231) en la quinta entrada dice:

En ciertas lenguas, categoría gramatical que expresa desarrollo interno de la acción verbal, según se concibe esta como durativa, perfecta o terminada, reiterativa, puntual, etc.

Mientras que el diccionario de María Moliner dice:

Componente del significado del verbo, con manifestación formal en ocasiones, por el cual se distinguen distintos tipos de acción: durativa, perfectiva, reiterativa, etc.

David Cohen (1993: 19) recoge multitud de definiciones entre las que podemos destacar que el aspecto es todo lo que no indica ni tiempo, ni modo, ni voz, ni persona o que

el aspecto es una categoría gramatical que expresa la representación que se hace el sujeto hablante del proceso expresado por el verbo (o por el nombre de acción), es decir, la representación de su duración, de su desarrollo o de su terminación (aspecto incoativo, durativo, resultativo, etc.), mientras que los tiempos, los modos y los auxiliares de tiempo expresan caracteres propios del proceso indicado por el verbo independientemente de la representación del proceso realizada por el sujeto hablante.

Vemos que el concepto de aspecto es de una enorme complejidad porque está sujeto a la percepción del sujeto hablante, aunque para nosotros de todas las acepciones que hemos encontrado la más adecuada es aquella en la que se concibe el aspecto como la percepción de la acción por parte de,l hablante, si ve la acción globalmente (aspecto perfectivo) o si la ve parcialmente sin importarle el principio y fin de la misma (aspecto imperfectivo).

Por lo tanto podemos explicar la categoría del aspecto en las lenguas eslavas con estas palabras:

W językach słowiańskich występuje kategoria gramatyczna zwana aspektem, która polega na tym, że każdy czasownik we wszystkich swoich formach czasowych i trybo- 
wych jest albo dokonany, albo niedokonany. Wyjątek stanowią tu nieliczne czasowniki dwuaspektowe lub takie, które w zależności od znaczenia mają aspekt dokonany lub niedokonany. Ta właściwość języków słowiańskich przeciwstawia je innym językom indoeuropejskim, na przykład łacinie i grece: w których dokonaność lub niedokonaność czynności wyraża się formą fleksyjną czasownika. (Śmiech, W. [1971] 6)

(En las lenguas eslavas destaca la categoría gramatical llamada aspecto, que consiste en que cada verbo, en todas sus formas temporales y modales, es o perfectivo o imperfectivo. Excepción hecha aquí de los escasos verbos que tienen los dos aspectos como los que, dependiendo del significado, tienen aspecto perfectivo o imperfectivo. La particularidad de las lenguas eslavas se opone a otras lenguas indoeuropeas, como, por ejemplo, el latín y el griego en las que la perfectividad o imperfectividad de la acción se aplica con la forma flexiva del verbo.)

Como hemos dicho antes, el aspecto destaca por encima de la categoría del tiempo, puesto que aparece en todas las formas verbales. Y esas marcas aspectuales se forman de las siguientes maneras:

2.1. Podemos añadir una serie de prefijos a la forma imperfectiva para obtener la perfectiva. Así en el verbo śpiewać (cantar) añadimos el prefijo za- (zaśpiewać) y conseguimos su par perfectivo. La serie de prefijos que podemos añadir es: $d o-$, od-, po-, prze-, za-, z-, u-, o-, na-.

Aunque a este método de formación de pares aspectuales verbales que es muy utilizado, se le añade la complicación de un segundo uso. Las lenguas eslavas también utilizar la prefijación verbal para crear verbos con nuevos significados como ocurre en el resto de las lenguas. De esta manera, nos encontramos el verbo escribir (pisaćl napisać) que crea su par perfectivo añadiendo un prefijo. Pero en el verbo inscribir (wpisywaćl wpisać) el prefijo $w$ - lleva una carga léxica y, por tanto, la marca morfológica del aspecto debe ser otra.

2.2. Otro procedimiento es añadirle a la forma perfectiva del verbo sufijos como -wać, -ować, -ywać e-iwać para formar el par imperfectivo. Como en la pareja verbal dawać / dać (dar).

2.3. Añadiendo el infijo - ie - en la raíz del par perfectivo para crear el imperfectivo. Como en zabrać/ zabierać (coger).

2.4. Utilizando la alternancia vocálica en la raíz con el siguiente esquema: $a-a$ / o- i. Ej. Wracać/ Wrócić (volver).

2.5. Usando un cambio de conjugación como se da en el verbo sprawdzaćl sprawdzić (verificar). En esta pareja el primer verbo, de valor imperfectivo, se conjuga por el tercer grupo y el segundo, por el segundo grupo.

2.6. Y, por último, utilizando verbos con raíces diferentes. Como mówićl powiedzieć (decir).

En cuanto al aspecto en español debemos destacar las palabras de Pedro Gomis:

Entre los muchos aspectos (incoativo, durativo, progresivo, perfectivo,...) que pueden expresarse en el verbo español y que, por lo tanto, pueden reforzar o modificar en todo o en parte su significado, los únicos que pueden manifestarse mediante las formas estrictamente temporales son el aspecto perfectivo y el imperfectivo. Los demás aspectos tendrán que indicarse en español mediante otros recursos lingüísticos: empleo de pronombres, perífrasis verbales, etc. [Gomis,P. (1998), 57] 
Aunque el aspecto en español no se reduce a expresar su perfectividad o su imperfectividad, sino que hay muchos más pares aspectuales. Sin embargo, se impone como prototípico este par de lo perfectivo-imperfectivo, porque es el único que aparecerá invariablemente en todos los verbos $\mathrm{y}$, en cambio, los demás van unidos o al significado o a determinadas estructuras.

\section{Equivalencias y diferencias de expresión entre los sistemas verbales}

$\mathrm{Si}$ nos centramos en los puntos que causan problemas a los estudiantes polacos, estos son:

elección de tiempo pretérito en una oración principal elección del tiempo en el estilo indirecto

el género en el verbo.

J. Pawlik nos da en su artículo sobre los pretéritos en polaco y el español una idea o, más bien, un aviso fundamental para el estudio de este tema. Un polaco de nivel medio, al entrar en contacto con lenguas extranjeras no eslavas hace la siguiente asociación: la oposición de aspectos tiene una correspondencia con los diversos tiempos pasados de otras lenguas. Así, en español, los alumnos polacos identifican frecuentemente el pasado de aspecto perfectivo polaco con los pretéritos indefinido y perfecto del español y el pasado de aspecto imperfectivo con el pretérito imperfecto español.

Pawlik expone esta cuestión con el siguiente ejemplo en el que aparece un complemento circunstancial que expresa duración:

\section{Czytałem przez dwie godziny.}

Leí durante dos horas.

Esta es la primera situación en que esa asociación rápida que hacen los alumnos no sirve, ya que un complemento de duración necesariamente irá con un verbo de aspecto imperfecto en polaco y, sin embargo, con el pretérito perfecto en español.

Otra situación que también diferencia los usos es que los verbos durativos polacos que indican continuidad aparecen expresados con aspecto imperfecto, en cambio, los verbos españoles se centran en la terminación de la acción independientemente de la duración de la misma.

Mój ojciec żył 70 lat.

Mi padre vivió 70 años.

La última diferencia que destaca Pawlik es la expresión de la resultatividad en los verbos. Mientras que esta siempre está clara con los verbos polacos, no es así en español. "No es posible determinar el grado de realización de una acción a partir de la forma misma del tiempo" en español. Aunque debemos mostrarnos en desacuerdo con respecto a este último punto. Este autor ha puesto ejemplos en los que da al pretérito perfecto un valor indiferente en cuanto a la resultatividad. 
Po potudniu pomalowatem dom.

Por la tarde pinté toda la casa.

Po potudniu malowałem dom.

Por la tarde pinté/estuve pintando la casa.

Puesto que un hablante español en las frases en las que usamos el pretérito perfecto simple sin marcadores temporales entiende que la acción está acabada y, aunque morfológicamente no aparece, que se la realizado hasta su conclusión. Así pues, en el otro ejemplo que da, lei el libro, no estamos de acuerdo en que haya dos interpretaciones: 1) lo acabé, 2) lo dejé sin acabar.

Esta misma manera de interpretación la plantea Nowikow con el caso que expone:

Fulano leyó varios libros, en el que expresa la ambigüedad a la hora de interpretar si leyó varios libros completamente o fragmentos de los mismos.

$X$ przeczytat wiele ksiażek $\neq X$ czytat wiele ksiażek

Aunque volvemos a señalar que el hablante español entiende la acción acabada y ejecutada completamente.

Por otro lado, otra idea que encontramos en el trabajo de Nowikow (2003: 197) y que deberemos tener en cuenta a la hora de elegir el pretérito español es que el uso de la forma de aspecto imperfectivo en polaco siempre expresará que la acción no está acabada, sea o no relevante, mientras que en castellano no tenemos esa información. Por ello cuestiona los estudios más recientes que presentan la oposición canté/ cantaba con criterios aspectuales. Al término pretérito se le relacionan los términos 'acción acabada', 'terminativo', 'perfectivo', 'puntual', ... y al pretérito imperfecto, 'acciones inacabadas', 'no terminativo', imperfectivo', 'duración', ... Esta oposición de los aspectos se ve matizada por la temporalidad.

Una segunda idea importante es que en las lenguas eslavas tanto las formas de aspecto perfectivo como las de aspecto imperfectivo pueden llevar complementos que expresen límite temporal, en cambio, es difícil que en español encontremos estos complementos con el pretérito imperfecto. Por tanto,

[...] una acción concluida como proceso tiene al mismo tiempo límite temporal. Es por eso por que en la mayoría de los casos a las formas del aspecto perfectivo en polaco

o en ruso les corresponden las formas del pretérito en español o en otras lenguas románicas. ${ }^{1}$ (Nowikow, 2003: 203, vol. 29)

$\mathrm{La} \pm$ conclusión procesal es un efecto secundario de la \pm delimitación temporal siempre condicionada por las propiedades semántico-gramaticales del texto.

Si nos fijamos en los ejemplos de los marcadores temporales que da este autor, en todos los ejemplos españoles dados con complementos temporales delimitadores se establece una correspondencia con el aspecto imperfectivo en polaco.

Ayer llovió todo el día.

Wczoraj padało przez caly dzień. 
Vivió diez años en Madrid.

Mieszkał przez dziesięć lat w Madricie.

No sé cuánto tiempo trabajó.

Nie wiem ile czasu (jak dlugo) pracowała.

Escuché música hasta las dos de la mañana.

Stuchatem muzyki do drugiej w nocy. (Nowikow, 2002: 181)

Así expone como conclusión que "los marcadores léxicos con valor limitativo no condicionan en polaco el empleo de las formas aspectualmente perfectivas", de ahí la no coincidencia completa del pretérito perfecto en español con el pasado de aspecto perfectivo en polaco. La coincidencia se da al darse la conclusión procesal que conlleva una limitación temporal, pero que, al revés, la limitación temporal no conlleva una conclusión procesal, no se da. Y esto lo expone con otro ejemplo:

Comí paella.

Necesariamente se desambigua en polaco ya que la primera opción de traducción, jadtem paelle,

informa de que la acción de comer ha terminado pero no ha concluido el proceso, porque queda paella en el plato. En cambio, con la segunda opción de traducción:

zjadtem paelle,

se sabe que la acción de comer ha terminado y se ha realizado hasta su conclusión porque ya no hay más paella.

En este último caso, para defender la interpretación que da Nowikow de la frase hay que tener en cuenta necesariamente la ausencia del artículo. Al no aparecer el artículo no se individualiza el objeto y, por tanto, no se puede cuantificar (PRADO 1999). Por consiguiente este ejemplo que no deja clara la conclusión procesal en español se ve matizado por el complemento; pero si el ejemplo hubiera presentado el artículo en su forma determinada o indeterminada la conclusión procesal estaría fuera de toda duda.

Debemos destacar que los problemas en un sentido y otro de aprendizaje, obviamente, no son los mismos. Por tanto, el problema con el que se encuentran los estudiantes españoles de polaco es la elección de la aspectualidad en el verbo principal, mientras que en la elección del tiempo no tienen casi problema, sea en una frase de estilo directo o de estilo indirecto. En cambio, para los estudiantes polacos de español el mayor problema será la elección de un tiempo pretérito en ambas situaciones.

A esto le tenemos que añadir una dificultad más, aunque pequeña, y es la del uso de los tiempos como absolutos o relativos. Mientras que en polaco la consecutio temporum es muy sencilla, no lo es tanto en español. En polaco la idea de anterioridad se expresa con el tiempo relativo en pasado independientemente del momento en que se sitúe la acción. La simultaneidad se expresa con el tiempo presente y la posterioridad con el futuro. Así en la expresión del verbo de la oración subordinada en la frase:

Maria mówi że kupiła ksiażkę.

María dice que ha comprado el libro. 
Maria powiedziała że kupiła ksiakę.

María dijo que había comprado el libro.

Ofrecerá dos posibilidades en función de la situación en el eje temporal de la acción, ya que si el verbo principal está en presente no hace falta cambiar el tiempo del mensaje. En cambio, si el verbo principal se sitúa en pasado debemos cambiarlo, aunque siga manteniendo la idea original. De todas maneras, este es un problema menor ya que, una vez dado el tiempo en el estilo directo, el cambio se produce siguiendo un esquema muy claro.

A. Presente de ind.; Pret. imperfecto de ind. > Pret. imperfecto de ind.;

B. Pret. perfecto simple; Pret. perfecto compuesto de ind.; Pret. pluscuamperfecto de ind. $>$ Pret. pluscuamperfecto de ind;

C. Futuro de ind.; Condicional simple $>$ Condicional simple;

D. Imperativo $>$ Pret. imperfecto de subj.

En cuanto al género en las formas verbales pretéritas debemos señalar brevemente que, aunque no supone un problema para los estudiantes que han aprendido alguna otra lengua no eslava antes, sí puede presentar alguna complicación en alumnos que adquieren primero un conocimiento rudimentario del español por inmersión y que después se acercan al aula para estudiarlo. Este es el caso de los polacos que vienen a España por motivos de trabajo. Por ello el profesor de español como lengua extranjera necesita conocer este rasgo del verbo polaco.

El verbo polaco no posee género en el tiempo presente, pero si en el pasado y en una de las formas para expresar el futuro. Así, en el singular en la primera persona aparecen dos formas: kupi-tem (masc.) / kupi-tam (fem.); en la segunda: kupi-teś (masc.) / kupi-laś (fem.); en la tercera persona: kupi-l (masc.) / kupi-la (fem.) / kupito (neutro). En plural la división por géneros cambia y nos encontramos la categoría de viril para los sujetos masculinos de persona y la categoría de no viril para el resto de sujetos (masculinos no personales e inanimados, femeninos y neutros). Así, en el plural en la primera persona tenemos: kupi-liśmy (viril) / kupi-byśmy (no viril); en la segunda: kupi-liście (viril) / kupi-lyście (no viril); en la tercera: kupi-li (viril) / kupi$\boldsymbol{b}$ (no viril). En el futuro aparece la tercera persona del singular o plural concordando con el sujeto en género junto con el verbo być (ser) conjugado en tiempo futuro. Por todo esto, podemos encontrarnos el caso de que algún estudiante asocie en el verbo la terminación -a en el pasado con el femenino igual que han hecho con los sustantivos. Así, en el pretérito imperfecto, especialmente, podemos ver que algún alumno dice *yo estabo, en vez de yo estaba por esta asociación. Este último dato no lo hemos encontrado reflejado en ningún estudio sino en nuestra experiencia docente, ya que se trata de un caso muy puntual. Son, pues, unas circunstancias de aprendizaje, como ya hemos señalado, muy específicas. 


\section{Conclusión}

El profesor de español como lengua extranjera ha de tener unos conocimientos básicos de las lenguas maternas de sus alumnos para poder enseñar de una manera más rápida y eficaz. En el caso de los alumnos polacos, los puntos conflictivos en el aprendizaje del verbo español serán la asociación de tiempos españoles y polacos por la noción de perfectividad, la relación de tiempos en el estilo indirecto y, como caso muy marginal, la ausencia de género en el sistema verbal español.

\section{Referencias bibliográficas}

COHEN, D. (1993): El aspecto verbal, Visor, Madrid.

GOMIS, P., SEGURA, L. (1998): Vademécum del verbo español, SGEL, Madrid.

MORA SÁNCHEZ, M.A. (1993): "Del texto a la gramática: sobre la enseñanza del estilo indirecto libre (EIL) en la clase de E/LE", Problemas y Métodos en la Enseñanza del Español como Lengua Extranjera, IV Congreso Internacional de ASELE, 381-390, http://cvc.cervantes.es/ensenanza/biblioteca_ele/asele/asele_iv.htm

NOWIKOW, W. (2002): "Acerca de la distinción entre los conceptos de [ \pm limitación temporal] y [ \pm conclusión procesal]", Romanica Cracoviensia, II, 177-186.

NOWIKOW, W. (2003): "Sobre las propiedades temporales y aspectuales: el pretérito en la construcción estar + gerundio", Studia Romanica Posnaniensia, XXIX, 197-204.

NOWIKOW, W. (2003): "«Terminativo» vs. «no terminativo»: entre oposiciones y ambigüedades tempo-aspectuales (el caso de los pretéritos)", Studia Romanica Posnaniensia, XXX, 123-129.

PAWLIK, J. (1995): "El aspecto y el sistema de los pretéritos del verbo en polaco y en español”, Estudios Hispánicos, IV, 87-96.

PRESA GONZÁLEZ, F. (2008): Gramática Polaca, Catédra, Madrid.

PRADO IBÁN, M M E. (1999): "El artículo: el/ un/ ausencia de artículo", Nuevas Perspectivas en la Enseñanza del Español como Lengua Extranjera, X Congreso Internacional de ASELE, 559-567, http://cvc.cervantes.es/ensenanza/biblioteca_ele/asele/asele_x.htm

RAE (2005): Diccionario de la Lengua Española, XXII edición, Espasa, Madrid. 